\title{
Serine protease inhibitors protect better than IL-10 and TGF- $\beta$ anti-inflammatory cytokines against mouse colitis when delivered by recombinant lactococci
}

Luis G Bermúdez-Humarán ${ }^{1,2 \dagger}$, Jean-Paul Motta ${ }^{3,4,5,6 \dagger}$, Camille Aubry ${ }^{1,2}$, Pascale Kharrat ${ }^{1,2}$, Laurence Rous-Martin ${ }^{3,4,5}$, Jean-Michel Sallenave ${ }^{7,8,9}$, Céline Deraison ${ }^{3,4,5}$, Nathalie Vergnolle ${ }^{3,4,5,9 \dagger}$ and Philippe Langella ${ }^{1,2^{*}}$

\begin{abstract}
Background: Different studies have described the successful use of recombinant lactic acid bacteria (recLAB) to deliver anti-inflammatory molecules at the mucosal level to treat Inflammatory Bowel Disease (IBD).

Methods: In order to identify the best strategy to treat IBD using recLAB, we compared the efficacy of different recombinant strains of Lactococcus lactis (the model LAB) secreting two types of anti-inflammatory molecules: cytokines (IL-10 and TGF- $\beta 1$ ) and serine protease inhibitors (Elafin and Secretory Leukocyte Protease Inhibitor: SLPI), using a dextran sulfate sodium (DSS)-induced mouse model of colitis.

Results: Our results show that oral administration of recombinant L. lactis strains expressing either IL-10 or TGF- $\beta 1$ display moderate anti-inflammatory effects in inflamed mice and only for some clinical parameters. In contrast, delivery of either serine protease inhibitors Elafin or SLPI by recLAB led to a significant reduction of intestinal inflammation for all clinical parameters tested. Since the best results were obtained with Elafin-producing L. lactis strain, we then tried to enhance Elafin expression and hence its delivery rate by producing it in a L. lactis mutant strain inactivated in its major housekeeping protease, HtrA. Strikingly, a higher reduction of intestinal inflammation in DSS-treated mice was observed with the Elafin-overproducing htrA strain suggesting a dose-dependent Elafin effect.
\end{abstract}

Conclusions: Altogether, these results strongly suggest that serine protease inhibitors are the most efficient anti-inflammatory molecules to be delivered by rec $L A B$ at the mucosal level for IBD treatment.

Keywords: Elafin, Proteases, Inflammation, Colitis, Inflammatory bowel disease, Lactococcus lactis, Probiotics

\section{Background}

Inflammatory bowel disease (IBD) is a group of chronic inflammatory disorders that cause inflammation of the digestive tract. The two major forms of IBD are Crohn's Disease (CD) and Ulcerative Colitis (UC) and they are characterized by an uncontrolled inflammatory response to lumenal content [1]. Despite the fact that several antiinflammatory molecules have been tested in preclinical and clinical models for IBD treatment, their therapeutic

\footnotetext{
*Correspondence: philippe.langella@jouy.inra.fr

${ }^{\dagger}$ Equal contributors

'INRA, Commensal and Probiotics-Host Interactions Laboratory, UMR 1319 Micalis, F-78350 Jouy-en-Josas, France

${ }^{2}$ AgroParisTech, UMR1319 Micalis, F-78350 Jouy-en-Josas, France

Full list of author information is available at the end of the article
}

potential and clinical application have been frequently hampered by different obstacles such as successful delivery or even toxic side-effects [1]. In the last 10 years, an increasing number of clinical and experimental studies have proven that probiotic bacteria may counteract the chronic inflammatory process. This effect is achieved by stabilizing the gut microbial environment and permeability barrier functions and by modulating the microbiota composition [2,3]. In addition, the successful use of food-grade Lactic Acid Bacteria (LAB) for the oral delivery of anti-inflammatory molecules to the inflamed intestine in preclinical experiments, as well as clinical trials have been reported [4-10]. This approach is based 
on the local synthesis and delivery of therapeutic molecules by viable recombinant $\mathrm{LAB}$ (recLAB) in situ.

The pioneer use of such recLAB for the prevention and treatment of experimental IBD was performed by Steidler et al. [11] who developed a recombinant strain of Lactococcus lactis (the LAB model) secreting biologically active anti-inflammatory cytokine IL-10. Interestingly, the authors showed that daily oral administration of $L$. lactis IL-10 in mice resulted in $\sim 50 \%$ reduction in dextran sulfate sodium (DSS)-induced colitis [11]. The beneficial effect of L. lactis IL-10 strain was dependent on the in situ secretion of IL-10 by recombinant live lactococci. Steidler et al. have then developed the first biocontainment system for L. lactis IL-10 strain to start the first human clinical study using it [12]. A phase I clinical trial was then conducted with this biocontained $L$. lactis IL-10 strain in Crohn's disease patients, showing that the containment strategy was effective [13]. Following this, a phase IIA trial was performed and a press release was published in 2009 revealing that all three primary endpoints have been met: i) safety and tolerability; ii) environmental containment and iii) assessment of biomarkers associated with the strain (data from ActoGeniX press release). Unfortunately, the clinical results did not reveal a statistically significant difference in mucosal healing with $L$. lactis IL-10 versus placebo. In view of these results, one can wonder whether IL-10 was the right choice of anti-inflammatory molecule to be delivered by recLAB. Other anti-inflammatory molecules to be delivered by recLAB should thus be tested.

Recent work has involved proteases and their endogenous inhibitors in the pathology of IBD [14-16]. Indeed, intestinal tissues from CD and UC patients showed elevated proteolytic activity $[14,15]$. This high proteolytic activity could be due to either upregulated protease expression, or decreased efficacy or expression of endogenous proteases inhibitors, or both. Transgenic mice producing human Elafin, an endogenous serine protease inhibitor found in the human gut, are protected from colitis in various mouse models of IBD [14]. We thus constructed recombinant $L$. lactis strains able to deliver Elafin at the mucosal level. We showed that the Elafin delivered by these recLAB prevents inflammation, accelerates mucosal healing and restores colon homeostasis in mice [17]. Although Elafin delivery at the mucosal surface by LAB was shown to efficiently reduce inflammatory signs in mouse colitis, one can wonder whether other protease inhibitors with a broader spectrum of inhibition might be as or more efficient. The Secretory Leukocyte Protease Inhibitor (SLPI, another serine protease inhibitor) inhibits the same elastases as Elafin (Elastase and Proteinase-3), but also inhibits Cathepsin G and trypsin, tryptase and chymase, major proteases contained in inflammatory cell granules. SLPI therefore appears as another possible attractive candidate to be delivered by LAB.

Besides the anti-inflammatory cytokine L. lactis IL-10, there is also Transforming Growth Factor- $\beta 1$ (TGF- $\beta$ ) which is an inhibitory cytokine recognized as a key regulator of immunological homeostasis and inflammatory responses [18]. Mice deficient for TGF- $\beta 1$ expression suffered from a more extensive autoimmune process with inflammatory infiltrates, involving multiple organs, including the intestine [19]. More important, despite the broad antiinflammatory and immune suppressive actions of TGF- $\beta 1$, to our knowledge, the potential anti-inflammatory effects of a mucosal delivery of this cytokine have not yet been compared to that of IL-10.

In order to identify the best strategy to treat IBD using recLAB as mucosal delivery carrier, we thus performed a comparison between $L$. lactis strains secreting between cytokines or serine protease inhibitors, using a DSS-induced colitis mouse model. We compared the efficacy of different recombinant strains of $L$. lactis secreting i) either IL-10 or TGF- $\beta 1$ as anti-inflammatory cytokines, and ii) either Elafin or SLPI as serine protease inhibitors. To further identify the best strategy to use recLAB, we constructed a recLAB strain inactivated in its major extracellular housekeeping protease, the high temperature requirement A (HtrA), which is supposed to produce higher quantities of the desired molecule [20]. Such approach aimed at determining the best vector, and defined whether a dose-dependent effect of the delivered molecule is important.

The most efficient protection against colitis was obtained by treatments with Elafin-producing L. lactis strain. This protective effect was enhanced with the Elafinoverproducing $h t r A$ strain, suggesting a dose-dependent effect of Elafin delivery. Altogether, these results showed that serine protease inhibitors are more efficient than anti-inflammatory cytokines as anti-inflammatory molecules to be delivered by recLAB at the mucosal level for IBD treatment.

\section{Results}

Production of anti-inflammatory cytokines and serine protease inhibitors by $L$. lactis

We constructed 4 different recombinant $L$. lactis strains that secrete i) either murine IL-10 (LL-IL-10) or murine TGF- $\beta$ (LL-TGF- $\beta$ ) as anti-inflammatory cytokines or ii) either human Elafin (L. lactis Elafin) or murine SLPI (L. lactis SLPI) as serine protease inhibitors. Production and secretion of these molecules were evaluated, and quantified when possible, by Western blot and ELISA assays, respectively. As shown in Figure 1A, a clear band was detected in the supernatant (S) fraction from induced LL-SLPI cultures at the expected size for mature SLPI (ie. without the $\mathrm{SP}_{\mathrm{Usp} 45}$ ); in these experimental conditions, no signal was detected in the 


\section{A) Murine Secreted Leukocyte Peptidase Inhibitor (SLPI)}

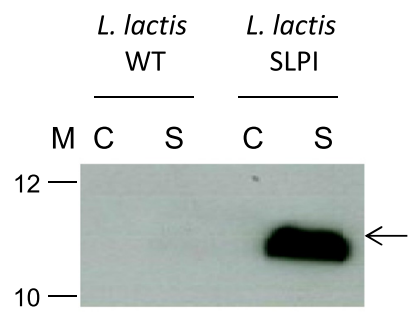

C) IL-10
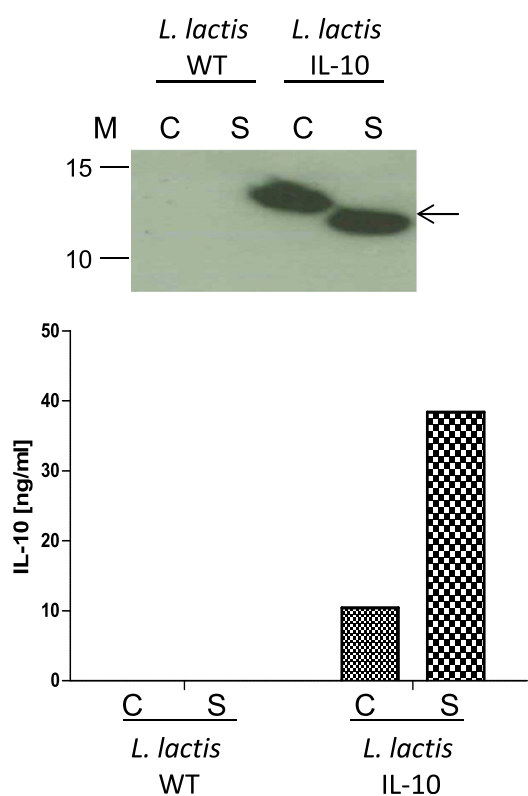

B) Elafin

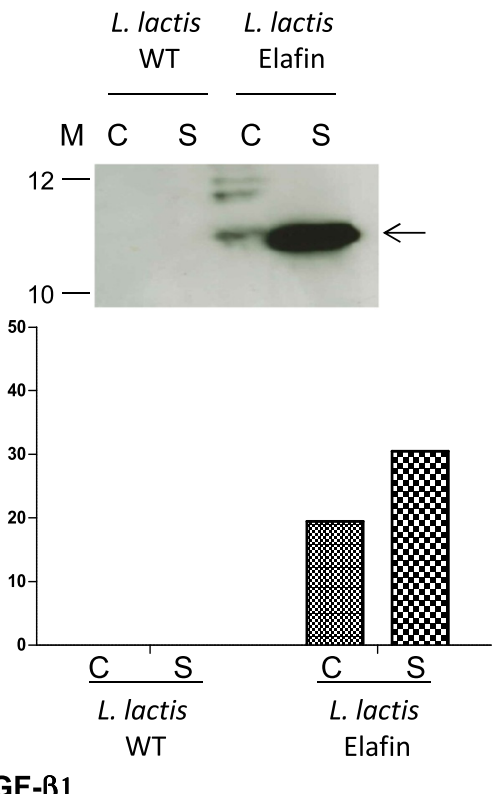

D) TGF- $\beta 1$

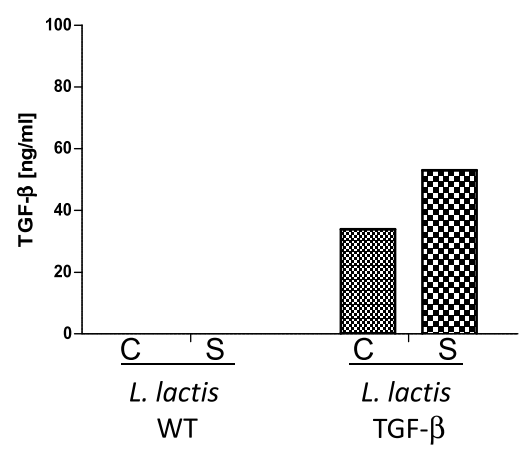

Figure 1 Characterization of recombinant Lactococcus lactis strains producing either anti-inflammatory molecules (IL-10 and TGF- $\beta 1$ ) or protease inhibitors (Elafin and SLPI). Protein production and secretion were analyzed by Western blotting (panels A, B and C) and ELISA (panel D) $1 \mathrm{~h}$ after induction with $1 \mathrm{ng} / \mathrm{ml}$ of nisin, the samples were then harvested at late exponential phase $\left(\sim \mathrm{OD}_{600}=0.8-1.0\right)$. Arrows indicate positions of mature proteins (eg. mSLPI panel A, human Elafin panel B, and mIL-10 panel C). Abbreviations: C, cell lysates; S, supernatant fraction; M, positions and sizes of molecular mass markers.

cell fraction (C) fraction suggesting an optimal secretion efficiency $(\sim 100 \%)$. Similar analysis of LL-Elafin strain resulted in different profiles in $\mathrm{C}$ and $\mathrm{S}$ samples (Figure 1B): i) two weak bands in the $\mathrm{C}$ fraction which likely correspond to either anomalous Elafin migration on SDS-PAGE or Elafin dimers and a weak band which slightly migrated higher than Elafin and which corresponds to $\mathrm{SP}_{\text {Usp45 }}$-Elafin precursor (preElafin) and ii) a clear single band in the $\mathrm{S}$ fraction at the expected size for mature Elafin. In addition, Elafin production and secretion by recombinant lactococci were quantified by ELISA and estimated at $\sim 35 \mathrm{ng} / \mathrm{ml}$ (Figure 1B).

Concerning IL-10 expression by LL-IL-10, our results reveal a good production and secretion of this cytokine by recombinant lactococci since more than $70 \%$ was found in $\mathrm{S}$ fraction (Figure 1C). These results were also validated by ELISA and estimated at $40 \mathrm{ng} / \mathrm{ml}$ (Figure 1C).

TGF- $\beta$ production by LL-TGF- $\beta$ strain was assessed only by ELISA and estimated at $50 \mathrm{ng} / \mathrm{ml}$ (Figure 1D). 
The results show that $L$. lactis is able to produce and efficiently secrete (secretion rate of $\sim 50 \%$ ) this cytokine.

The quantity of all recombinant molecules secreted from $\mathrm{LAB}$ was more or less in the same order of magnitude, insuring thereby the possibility to compare the efficiency of treatments with the different recLAB.

We should mention that none of the recombinant proteins was detected in samples obtained from $L$. lactis wild-type (WT) strain used as the negative control for Western Blot and ELISA assays (Figure 1A-D).

\section{Serine protease inhibitors-expressing L. lactis strains markedly reduced intestinal inflammation in a DSS-induced murine colitis model}

To define the best strategy to treat IBD using recLAB, we compared recombinant strains of $L$. lactis secreting the different anti-inflammatory candidates, using a DSSinduced murine colitis model.

Colitis was induced in C57BL/6 mice by addition of DSS $(5 \% \mathrm{w} / \mathrm{v})$ in drinking water for 7 days. This caused an acute inflammation characterized by increased macroscopic (Figure 2A) and microscopic (Figures 2B and 3) damage score, increased colon thickness (Figure 2C), prominent granulocyte infiltration (MPO activity, Figure 2D) and higher proteolytic activity (Figure 2E and F) in inflamed colonic tissues. Daily oral administrations of recombinant $L$. lactis secreting serine protease inhibitors resulted in a significant reduction of inflammation (decreased macroscopic and microscopic colonic damage scores, colon thickness and MPO activities), when compared to control mice (treated with either PBS or WT L. lactis strain). In addition, lower elastolytic activity was observed in colonic washes from mice receiving both LL-Elafin and LL-SLPI (Figure 2E). Trypsin-like activity was also significantly reduced in colonic washes from colitic mice that have received oral treatment with LL-Elafin (Figure 2F). Decreased inflammatory signs (damages score, wall thickness and MPO activity) were also observed in colonic tissues harvested from DSS-treated mice treated with L. lactis IL-10 (Figures $2 \mathrm{~A}, \mathrm{~B}$ and $\mathrm{D}$ and 3 ). Interestingly, the protective effects against colitis were higher in mice treated with the two L. lactis strains recombinant for serine protease inhibitors, than those treated with LL-IL-10 (macroscopic and microscopic score and colon thickness) (Figures 2A and B and 3).

Recombinant L. lactis strains expressing TGF$\beta$ displayed beneficial effects for MPO activities (Figure 2D) and histological scores (Figure 2B). However, this last, was not significantly different when compared to control mice. These results suggest that TGF- $\beta$ is a less efficient anti-inflammatory molecule when delivered by recombinant $L$. lactis to treat colitis.
Improving the anti-inflammatory properties of Elafin-producing L. lactis

As oral administration of serine protease inhibitorsproducing $L$. lactis strains, and more particularly LLElafin, was the most efficient strategy to inhibit DSSinduced colitis, we thus evaluated whether an improvement in the Elafin production and secretion by L. lactis could have an impact on its beneficial effects. We have previously reported that inactivation of the major $L$. lactis housekeeping protease, HtrA, allows high-level production and secretion of heterologous proteins $[20,21]$. We then established pSEC:hElafin plasmid in $L$. lactis htrA strain [22] to obtain L. lactis (htrA $\Delta$ ) Elafin and assessed Elafin production and secretion by Western blot (Figure 4A). In addition, Elafin secretion by $L$. lactis htrA $\triangle$ Elafin strain was quantified and compared with that of its counterpart L. lactis Elafin by ELISA and estimated at $\sim 55 \mathrm{ng} / \mathrm{ml}$ versus $37 \mathrm{ng} / \mathrm{ml}$ (Figure 4A). Since L. lactis htrA $\Delta$ strain has tendency to grow slower than WT L. lactis strain [21] we decided to compare growth curves (data not shown) of both strains and to determine elafin production per bacteria CFU (ng elafin/CFU/time) (Figure 4B). To compare the amounts of Elafin produced by recombinant $L$. lactis the results were normalized to a standard culture volume of $1 \mathrm{ml}$ of $1.0 \mathrm{OD}_{600}$ unit of culture (e.g. $\mathrm{OD}_{600}=$ $1=5 \times 10^{8} \mathrm{CFU}$ of L. lactis). As shown in Figure $4 \mathrm{~B}$, time-course experiments by ELISA comparing Elafin production by the two strains revealed that, despite its lower grow L. lactis htrA $\Delta$ strain produces higher levels of Elafin, compared to its counterpart L. lactis Elafin strain. This improvement in Elafin yields confirms the interest of L. lactis htrA strain to enhance production and secretion of human Elafin. We then compared the effects of these two strains in the DSS-induced murine colitis model. As shown in Figure $4 \mathrm{C}$, oral administration of mice with L. lactis (htrA $\Delta$ ) Elafin strain resulted in a better reduction of colonic damage scores, compared to the L. lactis Elafin strain. Treatment of mice with the $L$. lactis $h t r A \Delta$ Elafin strain was also very efficient to reduce proteolytic activity in inflamed colonic tissues: both elastolytic (Figure 4D) and trypsin-like activity (Figure 4E). Indeed, a better inhibition of colonic washes proteolytic activity was achieved with the L. lactis htrA $\Delta$ Elafin strain, compared to the LL-Elafin strain (Figure 4C-E). Altogether, these results suggest a dose-dependent effect of human Elafin when delivered in situ by recombinant $L$. lactis.

\section{Methods}

Bacterial strains and culture conditions

Escherichia coli TG1 [23] was grown in Luria-Bertani (Difco) medium at $37^{\circ} \mathrm{C}$ with vigorous agitation and both Lactococcus lactis MG1363 [24] and L. lactis NZ9000 [25] 
A)

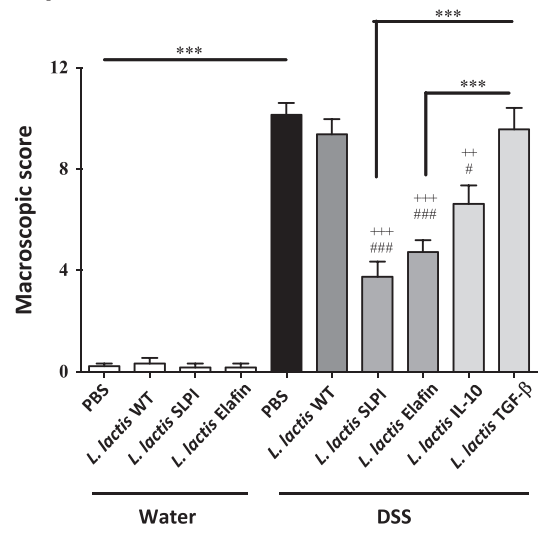

C)

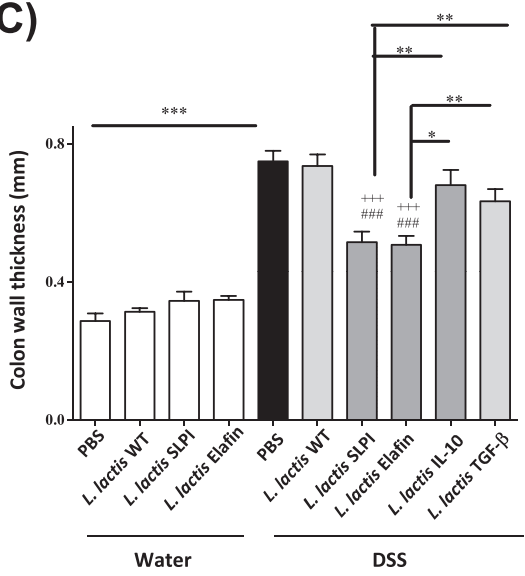

E)

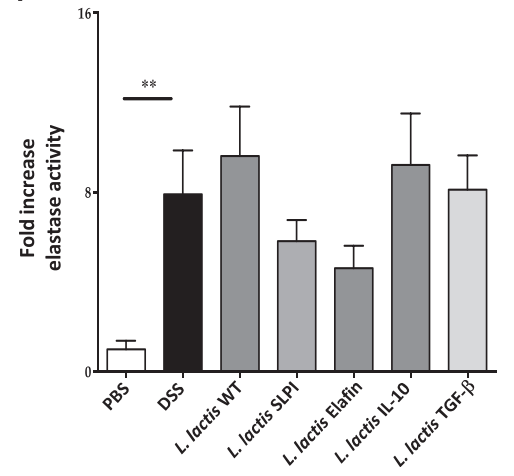

B)

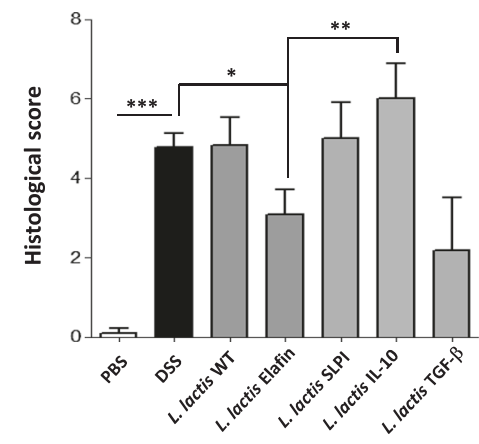

D)

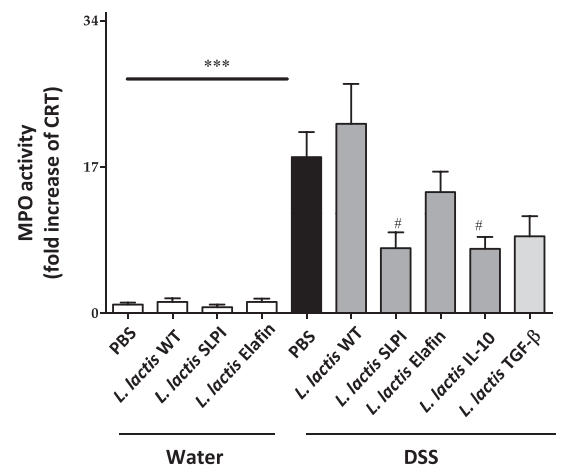

F)

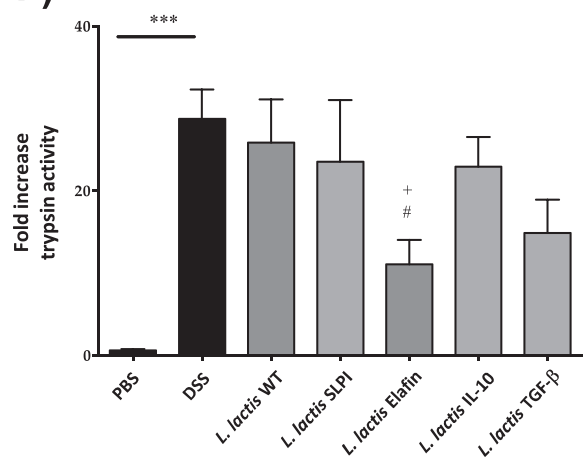

Figure 2 Effects of recombinant lactococci expressing 4 different molecules in a DSS-induced murine colitis model. C57BL/6 mice were given water or water containing 5\% DSS for 7 days and then received daily oral treatments for 7 days with either, vehicle (PBS), L. lactis wild-type (WT), Elafin-, SLPI-, IL-10- or TGF- $\beta$-expressing strains of L. lactis. (A-F). The macroscopic damage (A) and microscopic scores (B), colon wall thickness (C), and MPO activity (D) in mouse colon tissue are shown. Elastolytic (E) and trypsinolytic (F) activities were assessed in colonic lumenal washes. Significant differences is ${ }^{*},+, \#$ for $p<0.05,{ }^{* *},++, \# \#$ for $p<0.01{ }^{* * *},+++$,\#\# for $p<0.001$. + represents significant difference versus DSS and \# represents significant difference versus DSS + L.lactis WT. Data represents cumulative results of 4 independent experiments of $n=6-8$ mice per groups.

in M17 medium (Difco) supplemented with $0.5 \%$ glucose (GM17) at $30^{\circ} \mathrm{C}$. Antibiotics were used as follows: for $E$. coli $100 \mu \mathrm{g} / \mathrm{ml}$ of ampicillin or $15 \mu \mathrm{g} / \mathrm{ml}$ of chloramphenicol and for L. lactis $10 \mu \mathrm{g} / \mathrm{ml}$ of chloramphenicol.

\section{Construction of recombinant $L A B$ strains}

Gene encoding for murine TGF- $\beta 1$ (mTGF- $\beta 1)$ was amplified by PCR from plasmid pORF9-mTGF- $\beta 1$ (InvivoGen) using primers: forward mTGF- $\beta 1$ (CCAATGCATCAGCC 

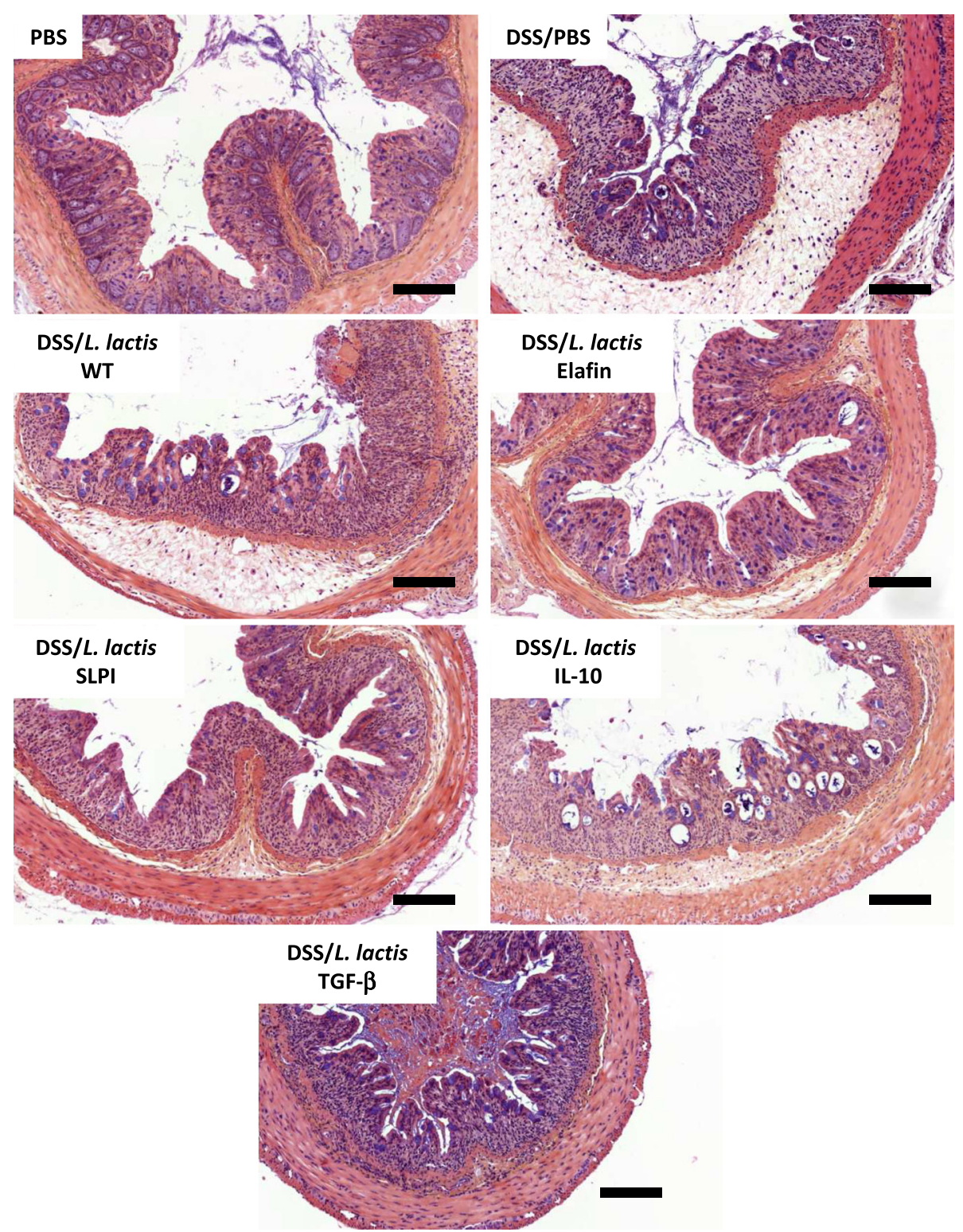

Figure 3 Representative images of H \& E staining on paraffin-wax-embedded sections of the colon of mice are presented. Scale bars represent $100 \mu \mathrm{m}$.

CTGGATACCAACTATTGC) and reverse mTGF- $\beta 1$ (GG ACTAGTCCTCAGCTGCACTTG CAGGAGC). Gene encoding for murine secretory leukocyte protease inhibitor (mSLPI) was amplified by PCR from plasmid pDK6mSLPI [26] using primers: forward mSLPI (CCAATG CATCAGGCAAAAATGATGCTATCAAAATCG) and reverse mSLPI (GG ACTAGTCCTCACATCGGGGGCAG GCAGACTTTCC). PCR products were subcloned into pCR:TOPO (Invitrogen, Table 1), recovered after digestion with NsiI/SpeI and cloned into a pSEC backbone purified from NsiI/NotI-cut pSEC:Nuc [27] (Table 1). Since all primers were designed to eliminate first genes codons encoding for the native signal peptide (SP), in the resulting plasmids (pSEC:mTGF- $\beta 1$ and pSEC:mSLPI), genes are fused in frame with a DNA fragment containing the ribosome binding site (RBS) and the SP of usp45 $\left(\mathrm{SP}_{\mathrm{Usp} 45}\right)$, the gene encoding Usp45, the predominant $L$. lactis-secreted protein [28]. In these plasmids, the expression is controlled by the inducible promoter $\mathrm{P}_{\text {nisA }}$, the activity of which depends upon the concentration of nisin used [29]. Plasmids were introduced in L. lactis NZ9000 strain to obtain LL-mTGF- $\beta 1$ and LL-mSLPI. 


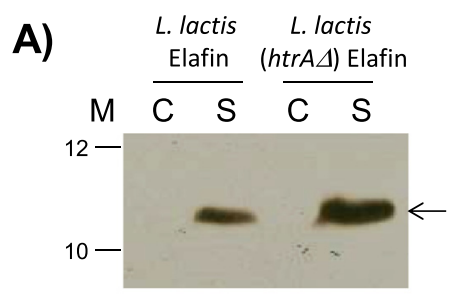

B)

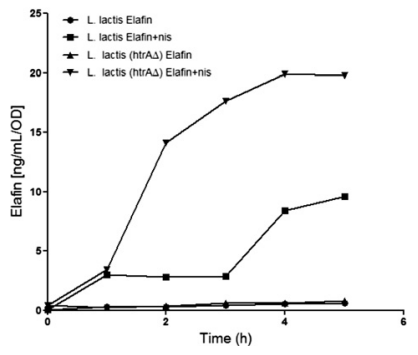

D)

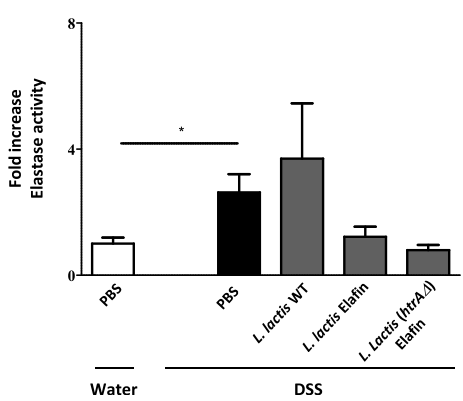

C)

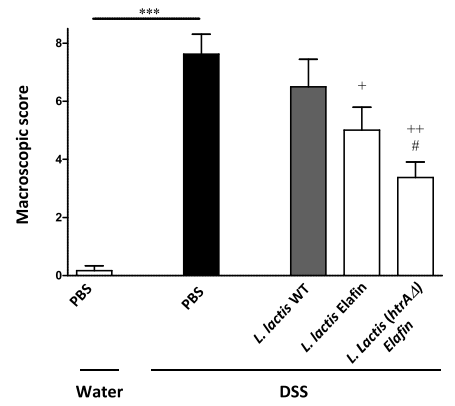

E)

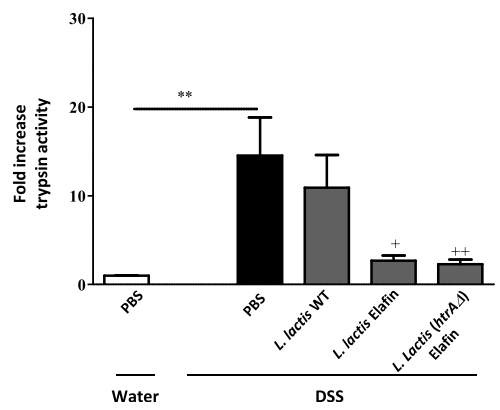

Figure 4 Enhancing Elafin production by L. lactis htrA mutant strain, as determined by Western blot (A) and ELISA (B) experiments, led to higher protective effects in a DSS-induced murine colitis model. C57BL/6 mice ( $n=8$ in each group) were given water or water containing $5 \%$ DSS for 7 days and then received daily oral treatments for 7 days with either, vehicle (PBS), L. lactis WT, Elafin-expressing L. lactis (L. lactis Elafin) or Elafin-expressing L. lactis-htra (L. lactis Elafin+). The macroscopic damage score (C) of the colon is shown. Elastolytic (D) and trypsinolytic (E) activities were assessed in colonic lumenal washes. Significant differences is ${ }^{*}$ for $p<0.05,{ }^{* *}$ for $p<0.01{ }^{* *}$ for $p<0.001$.

Recombinant L. lactis strains expressing either murine IL-10 (LL-mIL-10) or human Elafine (LL-hElafin) have been previously reported [17].

\section{Nisin induction, protein samples preparation and immunoblotting}

Recombinant L. lactis strains were grown to an optical density at $600 \mathrm{~nm}\left(\mathrm{OD}_{600}\right)$ of 0.6 , followed by induction with $1 \mathrm{ng}$ of nisin (SIGMA) per $\mathrm{ml}$ for $1 \mathrm{~h}$ as previously described [27]. L. lactis culture extraction and immunoblotting assays were performed as follows, using either murine SLPI (Santa Cruz Biotechnology, Inc., 10538), human Elafin (Santa Cruz Biotechnology, Inc., sc-20637) or murine IL-10 (Millipore, AB1492P) polyclonal antibodies. To determine SLPI, Elafin and IL-10 production, protein samples were prepared from $2 \mathrm{ml}$ of induced cultures. After centrifugation (5 min, $10,000 \mathrm{~g}$ ), the cell pellet $(\mathrm{C})$ and supernatant $(\mathrm{S})$ were treated separately.
The S was treated with $100 \mu \mathrm{l}$ of $100 \%$ trichloroacetic acid (TCA) to precipitate proteins and samples incubated for $10 \mathrm{~min}$ on ice. Proteins were recovered in PBS containing complete protease inhibitor cocktail tablets (Roche) after centrifugation at $4^{\circ} \mathrm{C}(10 \mathrm{~min}, 10,000 \mathrm{~g})$. The $\mathrm{C}$ was treated by cell lysis in lysis buffer (25\% sucrose, $1 \mathrm{mM}$ EDTA, $50 \mathrm{mM}$ Tris- $\mathrm{HCl} \mathrm{pH} 8.0$ and $10 \mathrm{mg} / \mathrm{ml}$ lysozyme) complemented with complete protease inhibitor cocktail tablets (Roche). Sodium dodecyl sulfate (SDS)-polyacrylamide gel electrophoresis, Western blotting, and immunodetection were performed following current protocol. The concentration of Elafin and IL10 produced by recombinant lactococci was also assessed in both $\mathrm{C}$ and $\mathrm{S}$ samples by enzyme-linked immuno-absorbent assays: RayBio ${ }^{\circ}$ human Trappin-2 ELISA Kit (RayBiotech, Inc. ELH-Trappin2-001) for Elafin and mouse IL-10 ELISA kit HRP (Mabtech, Sweden, 3431-1H-20). 
Table 1 Bacterial strains and plasmids used in this study

\begin{tabular}{|c|c|c|}
\hline $\begin{array}{l}\text { Strain or } \\
\text { plasmid }\end{array}$ & Characteristic $(\mathrm{s})^{a}$ & $\begin{array}{l}\text { Reference or } \\
\text { source }\end{array}$ \\
\hline & Reference or source & \\
\hline \multicolumn{3}{|l|}{ Strains } \\
\hline E. coli TG1 & supE, hsd, $\triangle 5$, thi, $\triangle$ (lac-proAB), F'(traD36 proAB-lacZ $\triangle M 15)$ & {$[23]$} \\
\hline L. lactis MG1363 & Wild type strain, plasmid free & {$[24]$} \\
\hline L. lactis NZ9000 & MG1363 (nisRK genes integrated into chromosome), plasmid free & {$[25]$} \\
\hline L. lactis NZ9000 htrA & NZ9000 carrying htrA disruption (double-crossover recombination) & {$[22]$} \\
\hline \multicolumn{3}{|l|}{ Plasmids } \\
\hline PCR-TOPO & $A p^{R}$, subloning TOPO vector & Invitrogen \\
\hline pORF9-mTGF- $\beta 1$ & $A p^{R}$, pORF9 plasmid carrying murine TGF- $\beta 1$ gene & InvivoGen \\
\hline pDK6-mSLPI & $\begin{array}{l}A^{R}{ }^{R}, p D K 6 \text { plasmid carrying full length murine secretory leukocyte protease inhibitor (SLPI) cDNA under the } \\
\text { control of the MCMV promoter }\end{array}$ & [26] \\
\hline pSEC:Nuc & $\begin{array}{l}\mathrm{Cm}^{R} \text {, pGK plasmid (a derivative from the broad host range plasmid pWV01) expressing a secreted form of the } \\
\text { staphylococcal nuclease (Nuc) under the control of } \mathrm{P}_{\text {nisA }} \text { pomoter }\end{array}$ & [27] \\
\hline pSEC:mIL-10 & $\mathrm{Cm}^{R}$, pGK plasmid expressing a secreted form of murine IL-10 cytokine under the control of $\mathrm{P}_{\text {nisA }}$ promoter & {$[17]$} \\
\hline pSEC:elafin & $\mathrm{Cm}^{R}$, pGK plasmid expressing a secreted form of human elafin antiprotease under the control of $P_{\text {nisA }}$ pomoter & {$[17]$} \\
\hline pSEC:mTGF- $\beta$ & $\mathrm{Cm}^{\mathrm{R}}$, pGK plasmid expressing a secreted form of murine TGF- $\beta$ cytokine under the control of $\mathrm{P}_{\text {nis }}$ pomoter & This study \\
\hline pSEC: mSLPI & $\mathrm{Cm}^{R}$, pGK plasmid expressing a secreted form of murine SLPI antiprotease under the control of $\mathrm{P}_{\text {nisA }}$ pomoter & This study \\
\hline
\end{tabular}

${ }^{\mathrm{a}}$ For strains, genotypic and phenotypic characteristics are given; for plasmids, plasmid and cloned-cassette characteristics are given.

TGF- $\beta$ production and secretion by $L$.lactis was assessed by ELISA as described above using a human/ mouse TGF- $\beta 1$ ELISA Ready-SET-Go kit (eBioscience, 88-8350).

Preparation of live bacterial inocula for gavage of animals RecLAB were grown as described above. Exponential growth cultures $(\mathrm{OD} 600=0.4-0.6)$ of L. lactis strains were treated for 1-2 hour with nisin ( $1 \mathrm{ng} / \mathrm{ml}$; Sigma) to induce recombinant protein expression. Recombinant strains of L. lactis were maintained with chloramphenicol $(10 \mu \mathrm{g} / \mathrm{ml})$. Bacteria were centrifuged after nisin induction, washed, and resuspended in corresponding volume of sterile PBS to get a final concentration of $5 \times 10^{9}$ colony forming units (CFU) for intragastric administration $(100 \mu \mathrm{l}$ per mouse).

C57BL/6 mice (6-8 weeks old) were obtained from Janvier (Le Genest Saint Isle, France). All mice were kept at room temperature under $12 \mathrm{~h}$ light/dark cycles, and had free access to food and water. All procedures were approved by the Animal Care Committee "Midi-Pyrénées" (MP/06/12/02/12).

\section{Induction of colitis and study design}

Colonic inflammation was induced by treatment with DSS (MP Biomedicals, Illkirch, France 160110), dissolved in drinking water ( $5 \%$ weight/volume). The animals were free to drink the DSS solution for 7 days. For the whole period of DSS exposure, mice were daily treated by intragastric gavage with $5 \times 10^{9}$ CFU (in $100 \mu \mathrm{L}$ PBS) of either wild-type (WT) or recombinant $L$. lactis strains or PBS $(100 \mu \mathrm{L})$. Body weight was measured daily after the induction of colitis. On day 7 after adding DSS to their drinking water, mice were sacrificed and colons were analyzed and harvested for measurement of inflammation parameters: macroscopic and microscopic damage score, bowel thickness, myeloperoxydase (MPO) activity as previously described $[14,17]$. Upon sacrifice, the entire colon was excised and $1 \mathrm{ml}$ PBS was instilled and washed twice through the lumen. Trypsin-like and elastase/PR-3-like activities were measured in those washes, using tosyl-Gly-ProArg- $p$-nitroanilide (150 $\mu \mathrm{M}$, Sigma) and MeO-succinylAla-Ala-Pro-Val- $\quad p$-nitroanilide $(100 \mu \mathrm{M}, \quad$ Sigma $)$ respectively as substrates. The change in absorbance at $405 \mathrm{~nm}$ was determined over 30 -minutes at $37^{\circ} \mathrm{C}$ with a microplate reader NOVOstarTM (BMG Labtech, Champigny-sur-Marne, France). Activity was compared to known standard dilution of trypsin from porcine pancreas (Sigma) or human NE (Sigma) [14,17].

\section{Statistics}

Data are presented as mean bars graph \pm SEM. Statistical analysis were performed using one-way analysis of variance (ANOVA), followed by suitable post hoc comparison tests (Bonferroni's or Dunnett's). A $P$ value less than 0.05 was considered significant. Graphic design and 
statistical analysis were performed using GraphPad Prism software version 5 for Windows.

\section{Discussion}

The treatment of IBD represents one of the major challenges of modern medicine as they concern several millions of people. Current therapies for IBD strongly need to be improved since a high percentage of patients (20$40 \%$ ) are resistant to any forms of treatments [30]. Moreover, severe side-effects and high costs are frequently associated to the currently available drugs (e.g. glucocorticoids and monoclonal antibody therapies).

Over the past 10 years, there has been increasing interest in the use of LAB as oral delivery vectors $[4,7]$. More particularly, the use of recombinant $L$. lactis secreting IL-10 for the prevention and treatment of colitis in different mouse models has been largely studied [11]. To date, two human clinical trials have been carried out with a biologically contained-derivative LAB (ie. lactic acid bacteria) secreting IL-10 [12]. The first study was performed in the Netherlands and assessed LAB IL-10 effects in Crohn's disease patients. The results of this small phase I human trial, showed positive effects concerning biological containment, safety and tolerability [13]. In the second study (a phase IIa human clinical trial), ActoGenix (a company which develops genetically modified L. lactis for mucosal delivery of therapeutic molecules), assessed the effects of LAB IL-10 in subjects with moderately active ulcerative colitis. Unfortunately, although the results confirmed the suitability of the applied containment system in humans, no significant clinical effects were observed in this study (ActoGenix press release published at the end of 2009). This limited efficacy could be explained either by the fact that IL-10 is not the best molecule of choice to be used in a recLAB system, or by the fact that the IL-10 quantities delivered were not sufficient to be efficient in the intestinal environment.

The choice of IL-10 as a molecule to be delivered by LAB is supported by the anti-inflammatory effects of this cytokine [31]. As a matter of fact, mice deficient for IL-10 develop spontaneous colitis [32]. However, while systemic treatments with recombinant IL-10 are safe and well tolerated, they have a low therapeutic efficacy compared with placebo [33]. This suggests that pharmacodynamics of IL-10 are subtle and potentially needs a sustained and more mucosa-focused delivery to be efficient against colitis. Indeed, sustained delivery of IL-10 through adenovirus-based strategy showed better results in animal models [34,35], whether the colitis was induced by IL-10 deficiency or by chemicals. However, discrepancy exists on the fact that adenovirus-based IL-10 systemic delivery might not be able to reduce established colitis [36]. Local delivery of IL-10, through intracolonic administration of an adenovirus expressing IL-10 was able to reduce colitis in IL-10-deficient mice [37]. However, no study has investigated whether this mucosal delivery of IL-10 was efficient against colitis involving other mediators than only IL-10 deficiency. This approach of using LAB as vectors for IL-10 mucosal delivery [11], ensure a sustained and local (mucosal surface) delivery. However, IL-10 is released within the lumen, and might not be able to penetrate deep into the tissues and to exert its anti-inflammatory properties within mucosal tissues. IL-10 has broad immunoregulatory activity, acting to suppress intestinal inflammation on several levels. It inhibits $\mathrm{T}_{\mathrm{H}} 1$ lymphocyte differentiation; it diminishes antigen presentation and IL-12 release, and promotes differentiation and activity of regulatory $\mathrm{T}$ cells $[31,38]$. All these activities are related to actions on immune cells present in the lamina propria, not at the mucosal surface. Taken together, all these facts argue against considering IL-10 as a molecule of choice to be delivered by recLAB, and indeed, we demonstrated that treatments of colitic mice with IL-10 recLAB was not efficient on most parameters of intestinal inflammation. We cannot discard also that the minor effect observed with the LL-IL-10 strain was due to our murine colitis model. Indeed, acute colitis models induced by DSS are characterized by massive epithelial damage and it has been well established that IL-10 gave the best results in chronic colitis models that are immunological driven.

TGF- $\beta 1$, another anti-inflammatory cytokine that could recapitulate some of the effects of IL-10 on immune cells, but that could also exert protective effects on intestinal epithelial cells, at the mucosal surface, appeared as a better candidate to be delivered by recLAB. Like IL-10, TGF- $\beta 1$ exerts multiple actions on immune cells (regulatory $\mathrm{T}$ cells, Th17 cells, monocytes, macrophages) and is also protective in intestinal epithelial cells. Beck et al. have shown that TGF- $\beta 1$ is required for intestinal mucosal healing, and strongly decreases the epithelial susceptibility to injury [39]. TGF- $\beta 1$ also inhibits intestinal barrier disruption, by a direct effect on intestinal epithelial cells $[40,41]$. Thus, we constructed TGF- $\beta 1$ recombinant $L$. lactis strain and tested this treatment against DSS-induced colitis. Like for IL-10 recombinant LAB, LL-TGF- $\beta$ oral treatments were poorly effective against colitis. Such treatment only reduced granulocytes infiltration (Figure 2D), but not the other parameters of inflammation. Here again, one can wonder if TGF- $\beta 1$ is the molecule of choice to be delivered by $L A B$, and could exert its anti-inflammatory effects, once released in the lumen. Therefore, we tested the effects of a completely different family of anti-inflammatory molecules, which are released by the intestinal epithelium, and at the mucosal surface: the serine protease inhibitors. Both Elafin and SLPI, when delivered by L. lactis 
and used as oral treatments, strongly inhibited DSSinduced colitis and were as effective (Figure 2). These serine protease inhibitors have different spectra of inhibition, SLPI being able to inhibit Cathepsin G and a number of trypsin like proteases (trypsin, tryptase and chymase), in addition to Elastase and Proteinase-3, also inhibited by Elafin $[42,43]$. This suggests that the sole inhibition of Elastase and Proteinase- 3 is sufficient to obtain strong anti-inflammatory effects. Cathepsin G and trypsin-like inhibition should not provide additional protective effects. Treatments with recombinant $L$. lactis strains delivering either SLPI or Elafin were both more efficient to reduce signs of colitis, than treatments with anti-inflammatory cytokines recLAB. Endogenous protease inhibitors released physiologically by the intestinal epithelium thus appear as better molecules to be delivered by L. lactis, to generate potent therapeutic option. Indeed, these two protease inhibitors are pleiotropic in their role as guardian of mucosal surfaces. They not only inhibit the effects of proteases released by inflammatory cells, but they also inhibit pro-inflammatory transcription factors (AP-1, NF-kB), restore barrier functions, and exert antimicrobial activity [17,26,43,44]. Released by LAB in the lumen, Elafin and SLPI can potentially exert their anti-inflammatory biological functions more easily than anti-inflammatory cytokines. In addition, Elafin and SLPI might impact on the microbiota composition, due to their antimicrobial activity, might not be negligible. Such role could add to the protective effects they might have on the host [43].

An important point to consider when using the recLAB technology is the quantity of protein of interest that is delivered at the mucosal surface. Here, we showed that treatment with $L$. lactis strain inactivated in the main extracellular protease and thus able to release more protein of interest (Elafin), is more efficient at reducing colitis. Therefore, the recLAB technology could be improved by using such $L$. lactis mutant. The quantity of protein released at the mucosal surface could also depend on the time the bacteria will survive in the gastrointestinal tract (GIT). One of the hypotheses for the lack of effect of treatment with $L$. lactis IL-10 in clinical trials, was the fact that this bacterium has a weak resistance to the gastrointestinal environment [45]. Although our experiments were performed in mice and not in human like the clinical trials, we have observed that even with L. lactis as a vector, serine protease inhibitors were more potent antiinflammatory molecules than IL-10 or TGF- $\beta 1$. Nonetheless, other $\mathrm{LAB}$ vectors more persistent in the GIT (as lactobacilli) to deliver the proteins of interest should be tested to improve the kinetics and associated quantity of protein delivered at mucosal surfaces.

In conclusion, we have observed that the use of L. lactis strains expressing anti-proteases (Elafin or SLPI) is more efficient than the use of either LL-IL-10 or LLTGF- $\beta$ in this colitis model, to decrease intestinal inflammation. This differential effect could be explained by the different anti-inflammatory functions of these classes of molecules, and the fact that $L$. lactis vector delivery might be more appropriate with mucosal surface proteins such as protease inhibitors, than proteins exerting most of their effects in the lamina propria, such as anti-inflammatory cytokines. These results strongly suggest that the administration of recombinant $L$. lactis strains expressing serine protease inhibitors would raise more interest than the L. lactis IL-10 recombinant approach for IBD treatment.

\section{Competing interests}

The authors declare that they have no competing interests.

\section{Authors' contributions}

LGBH, JPM, CD, NV and PL designed all the experiments. LGBH, JPM, CA, PK, $L R M, C D$ and JMS performed the experiments. $L G B H$ and PL wrote the manuscript. JPM, JMS, CD and NV corrected the manuscript. All authors read and approved the final manuscript.

\section{Acknowledgements}

The work was supported by the Agence Nationale de la Recherche (ANR PPAIN R0809688 to NV, and ANR-12-BSV1-0030-01 to NV and PL). JPM was supported by a studentship from INSERM/ Région Midi-Pyrénées, and by Ph. $D$ thesis awards from GREMI (Group for Research and studies on Mediators of Inflammation) and CECED (Digestive epithelial cells study group). CA was supported by the grant ANR-12-BSV1-0030-01.

\section{Author details}

${ }^{1}$ INRA, Commensal and Probiotics-Host Interactions Laboratory, UMR 1319 Micalis, F-78350 Jouy-en-Josas, France. ${ }^{2}$ AgroParisTech, UMR1319 Micalis, F-78350 Jouy-en-Josas, France. ${ }^{3}$ Inserm, U1043, Toulouse F-31300, France. ${ }^{4}$ CNRS, U5282, Toulouse F-31300, France. ${ }^{5}$ Université de Toulouse, UPS, Centre de Physiopathologie de Toulouse Purpan (CPTP), Toulouse F-31300, France. ${ }^{6}$ Department of Biological Sciences, Faculty of Science, University of Calgary, Calgary, Alberta, Canada. ${ }^{7}$ INSERM U874, Institut Pasteur, 25 rue du Dr Roux, 75015 Paris, France. ${ }^{8}$ INSERM U1152, Faculté de Médecine site Bichat, Université Paris Diderot, 16, rue Henri Huchard, 75018 Paris, France. ${ }^{9}$ Université Sorbonne Paris Cité, Université Paris Diderot, rue du Dr Roux, 75015 Paris, France.

Received: 28 August 2014 Accepted: 23 January 2015

Published online: 26 February 2015

\section{References}

1. Khor B, Gardet A, Xavier RJ. Genetics and pathogenesis of inflammatory bowel disease. Nature. 2011:474:307-17.

2. Veiga P, Gallini CA, Beal C, Michaud M, Delaney ML, DuBois A, et al. Bifidobacterium animalis subsp. lactis fermented milk product reduces inflammation by altering a niche for colitogenic microbes. Proc Natl Acad Sci U S A. 2010;107:18132-7.

3. McNulty NP, Yatsunenko T, Hsiao A, Faith JJ, Muegge BD, Goodman AL, et al. The impact of a consortium of fermented milk strains on the gut microbiome of gnotobiotic mice and monozygotic twins. Sci Transl Med. 2011;3:106ra106.

4. Bermúdez-Humarán LG, Aubry C, Motta JP, Deraison C, Steidler L, Vergnolle $\mathrm{N}$, et al. Engineering lactococci and lactobacilli for human health. Curr Opin Microbiol. 2013;16(3):278-83.

5. Bermudez Humaran LG, Kharrat P, Chatel JM, Langella P. Lactococci and lactobacilli as mucosal delivery vectors for therapeutic proteins and DNA vaccines. Microb Cell Fact. 2011;10 Suppl 1:S4.

6. LeBlanc JG, Aubry C, Cortes Perez NG, De Moreno De LeBlanc A, Vergnolle $\mathrm{N}$, Langella $\mathrm{P}$, et al. Mucosal targeting of therapeutic molecules using 
genetically modified lactic acid bacteria: an update. FEMS Microbiol Lett. 2013;344:1-9.

7. Wells JM, Mercenier A. Mucosal delivery of therapeutic and prophylactic molecules using lactic acid bacteria. Nat Rev Microbiol. 2008;6:349-62.

8. Steidler L, Rottiers P, Coulie B. Actobiotics as a novel method for cytokine delivery. Ann N Y Acad Sci. 2009:1182:135-45.

9. Steidler L, Rottiers P. Therapeutic drug delivery by genetically modified Lactococcus lactis. Ann N Y Acad Sci. 2006;1072:176-86.

10. Limaye SA, Haddad RI, Cilli F, Sonis ST, Colevas AD, Brennan MT, et al. Phase $1 \mathrm{~b}$, multicenter, single blinded, placebo-controlled, sequential dose escalation study to assess the safety and tolerability of topically applied AG013 in subjects with locally advanced head and neck cancer receiving induction chemotherapy. Cancer. 2013;119:4268-76.

11. Steidler L, Hans W, Schotte L, Neirynck S, Obermeier F, Falk W, et al. Treatment of murine colitis by Lactococcus lactis secreting interleukin-10 Science. 2000;289:1352-5.

12. Steidler L, Neirynck S, Huyghebaert N, Snoeck V, Vermeire A, Goddeeris B, et al. Biological containment of genetically modified Lactococcus lactis for intestinal delivery of human interleukin 10. Nat Biotechnol. 2003;21:785-9.

13. Braat $H$, Rottiers $P$, Hommes DW, Huyghebaert N, Remaut E, Remon JP, et al. A phase I trial with transgenic bacteria expressing interleukin-10 in Crohn's disease. Clin Gastroenterol Hepatol. 2006;4:754-9.

14. Motta JP, Magne L, Descamps D, Rolland C, Squarzoni-Dale C, Rousset P, et al. Modifying the protease, antiprotease pattern by elafin overexpression protects mice from colitis. Gastroenterology. 2011;140:1272-82.

15. Cenac N, Andrews CN, Holzhausen M, Chapman K, Cottrell G, AndradeGordon $\mathrm{P}$, et al. Role for protease activity in visceral pain in irritable bowel syndrome. J Clin Invest. 2007;117:636-47.

16. Hyun E, Andrade-Gordon P, Steinhoff M, Vergnolle N. Protease-activated receptor-2 activation: a major actor in intestinal inflammation. Gut. 2008:57:1222-9.

17. Motta JP, Bermudez Humaran LG, Deraison C, Martin L, Rolland C, Rousset P, et al. Food-grade bacteria expressing elafin protect against inflammation and restore colon homeostasis. Sci Transl Med. 2012:4:158ra144.

18. Hamady ZZ, Scott N, Farrar MD, Wadhwa M, Dilger P, Whitehead TR, et al. Treatment of colitis with a commensal gut bacterium engineered to secrete human TGF-beta1 under the control of dietary xylan 1. Inflamm Bowel Dis. 2011;17:1925-35.

19. Monteleone G, Pallone F, MacDonald TT. Smad7 in TGF-beta-mediated negative regulation of gut inflammation. Trends Immunol. 2004:25:513-7.

20. Miyoshi A, Poquet I, Azevedo V, Commissaire J, Bermudez-Humaran L, Domakova E, et al. Controlled production of stable heterologous proteins in Lactococcus lactis. Appl Environ Microbiol. 2002;68:3141-6.

21. Poquet I, Saint V, Seznec E, Simoes N, Bolotin A, Gruss A. HtrA is the unique surface housekeeping protease in Lactococcus lactis and is required for natural protein processing. Mol Microbiol. 2000;35:1042-51.

22. Rigoulay C, Poquet I, Madsen SM, Gruss A. Expression of the Staphylococcus aureus surface proteins $\operatorname{Htr} A(1)$ and $\operatorname{Htr} A(2)$ in Lactococcus lactis. Fems Microbiol Lett. 2004;237:279-88.

23. Gibson TJ. Ph.D. Thesis. Cambridge, England: University of Cambridge; 1984.

24. Gasson MJ. Plasmid complements of Streptococcus lactis NCDO 712 and other lactic streptococci after protoplast-induced curing. J Bacteriol. 1983:154:1-9.

25. Kuipers OP, de Ruyter PGGA, Kleerebezem M, de Vos WM. Quorum sensingcontrolled gene expression in lactic acid bacteria. J Biotechnol. 1998;64:15-21.

26. Henriksen PA, Hitt M, Xing Z, Wang J, Haslett C, Riemersma RA, et al. Adenoviral gene delivery of elafin and secretory leukocyte protease inhibitor attenuates NF-kappa B-dependent inflammatory responses of human endothelial cells and macrophages to atherogenic stimuli. J Immunol. 2004;172:4535-44.

27. Bermudez-Humaran LG, Langella P, Commissaire J, Gilbert S, Le Loir Y, $L$ 'Haridon R, et al. Controlled intra- or extracellular production of staphylococcal nuclease and ovine omega interferon in Lactococcus lactis. FEMS Microbiol Lett. 2003;224:307-13.

28. Vanasseldonk M, Rutten G, Oteman M, Siezen RJ, Devos WM, Simons G. Cloning of Usp45, a gene encoding a secreted protein from lactococcuslactis subsp lactis Mg1363. Gene. 1990;95:155-60.

29. de Ruyter PG, Kuipers OP, de Vos WM. Controlled gene expression systems for Lactococcus lactis with the food-grade inducer nisin. Appl Environ Microbiol. 1996:62:3662-7.
30. Triantafillidis JK, Merikas E, Georgopoulos F. Current and emerging drugs for the treatment of inflammatory bowel disease. Drug Des Devel Ther. 2011;5:185-210.

31. Moore KW, de Waal MR, Coffman RL, O'Garra A. Interleukin-10 and the interleukin-10 receptor. Annu Rev Immunol. 2001;19:683-765.

32. Kuhn R, Lohler J, Rennick D, Rajewsky K, Muller W. Interleukin-10-deficient mice develop chronic enterocolitis. Cell. 1993;75:263-74.

33. Fedorak RN, Gangl A, Elson CO, Rutgeerts P, Schreiber S, Wild G, et al. Recombinant human interleukin 10 in the treatment of patients with mild to moderately active Crohn's disease. The interleukin 10 inflammatory bowel disease cooperative study group. Gastroenterology. 2000;119:1473-82.

34. Lindsay JO, Ciesielski CJ, Scheinin T, Hodgson HJ, Brennan FM. The prevention and treatment of murine colitis using gene therapy with adenoviral vectors encoding IL-10. J Immunol. 2001;166:7625-33.

35. Lindsay J, van Montfrans C, Brennan F, van Deventer S, Drillenburg P, Hodgson H, et al. IL-10 gene therapy prevents TNBS-induced colitis. Gene Ther. 2002;9:1715-21.

36. Barbara G, Xing Z, Hogaboam CM, Gauldie J, Collins SM. Interleukin 10 gene transfer prevents experimental colitis in rats. Gut. 2000;46:344-9.

37. Lindsay JO, Ciesielski CJ, Scheinin T, Brennan FM, Hodgson HJ. Local delivery of adenoviral vectors encoding murine interleukin 10 induces colonic interleukin 10 production and is therapeutic for murine colitis. Gut. 2003;52:981-7.

38. Groux H, O'Garra A, Bigler M, Rouleau M, Antonenko S, de Vries JE, et al. A CD4+ T-cell subset inhibits antigen-specific T-cell responses and prevents colitis. Nature. 1997:389:737-42.

39. Beck PL, Rosenberg IM, Xavier RJ, Koh T, Wong JF, Podolsky DK. Transforming growth factor-beta mediates intestinal healing and susceptibility to injury in vitro and in vivo through epithelial cells. Am J Pathol. 2003;162:597-608.

40. Roche JK, Martins CA, Cosme R, Fayer R, Guerrant RL. Transforming growth factor beta1 ameliorates intestinal epithelial barrier disruption by Cryptosporidium parvum in vitro in the absence of mucosal T lymphocytes. Infect Immun. 2000;68:5635-44.

41. Howe KL, Reardon C, Wang A, Nazli A, McKay DM. Transforming growth factor-beta regulation of epithelial tight junction proteins enhances barrier function and blocks enterohemorrhagic Escherichia coli 0157:H7-induced increased permeability. Am J Pathol. 2005;167:1587-97.

42. Moreau T, Baranger K, Dade S, Dallet-Choisy S, Guyot N, Zani ML. Multifaceted roles of human elafin and secretory leukocyte proteinase inhibitor (SLPI), two serine protease inhibitors of the chelonianin family. Biochimie. 2008;90:284-95.

43. Sallenave JM, Silva A. Characterization and gene sequence of the precursor of elafin, an elastase-specific inhibitor in bronchial secretions. Am J Respir Cell Mol Biol. 1993;8:439-45.

44. Butler MW, Robertson I, Greene CM, O'Neill SJ, Taggart CC, McElvaney NG. Elafin prevents lipopolysaccharide-induced AP-1 and NF-kappaB activation via an effect on the ubiquitin-proteasome pathway. J Biol Chem. 2006;281:34730-5.

45. Watterlot L, Meyrand M, Gaide N, Kharrat P, Blugeon S, Gratadoux JJ, et al. Variations of $\mathrm{N}$-acetylation level of peptidoglycan do not influence persistence of Lactococcus lactis in the gastrointestinal tract. Int J Food Microbiol. 2010;144:29-34.

\section{Submit your next manuscript to BioMed Central and take full advantage of:}

- Convenient online submission

- Thorough peer review

- No space constraints or color figure charges

- Immediate publication on acceptance

- Inclusion in PubMed, CAS, Scopus and Google Scholar

- Research which is freely available for redistribution 